\title{
METABOLIC PROFILE TESTING IN DAIRY HERDS: WRONG ANSWER OR WRONG QUESTION?
}

\author{
Eicher $R$ \\ Div. Population Medicine, Dept. Clinical Vet. Med., University of Berne. Wanner M, Inst. Animal Nutrition, \\ University of Zurich. Rüsch P, Federal Veterinary Office, Switzerland
}

Metabolic profile testing (MPT) in dairy herds has been proposed as a diagnostic tool for the analysis of reproductive and metabolic or nutritional disorders. Results of such analyses have been contradictory. The goals of this study are to discuss some factors potentially influencing the interpretation of MPT results and to propose guidelines for its realisation. Specific objectives were 1. to discuss which metabolites can be used for the purpose of diagnosing disorders and which metabolites yield no useful information in healthy cattle, 2. to analyze the distribution of metabolites and the consequences of these distributions for chosing reference values, 3. to analyze the influence of the stage of lactation on the variability of chosen metabolites and the consequences of this variability on the strategy of making and interpretation of MPT, 4. the influence of season of testing, and 5. the influence of the site of punction on chosen metabolites.

Samples were taken in 36 herds for a total of 1787 samples. Sampling was performed 4 times during one year (2 samples each during grazing season and winter feeding period). Urine and blood samples were taken from milking cows between $9 \mathrm{~h}$ and $11 \mathrm{~h}$ in the morning. Cows were chosen in order to have a representative distribution across lactation stage. Mean sample size per herd was $n=11$ $(\mathrm{SD}=4)$. Generally, samples were taken through punction of the jugular vein. In several herds, however, punction of vena caudalis and/or vena epigastrica superficialis was also performed. Exploration of the data was performed with histograms and X-Y plots of the metabolites against days postpartum. Differences between seasons and sites of punction were shown as box plots and statistically tested according to the distribution with parametric or non-parametric tests.

There were typically different distributions among metabolites. Glucose, urea, cholesterol, calcium, phosphorus, and magnesium were normally distributed, whereas NEFA, ß-hydroxybutyrate, liver enzymes as well as urinary sodium, potassium, and magnesium were heavily skewed. Some metabolites showed characteristic patterns depending on days postpartum (glucose, NEFA, B-hydroxybutyrate, cholesterol), whereas other did not show such patterns (urea, calcium, phosphorus, magnesium, sodium, liver enzymes). Relevant differences between seasons were found for phosphorus, magnesium, urea, and potassium. Differences between veins were found for glucose, ß-hydroxybutyrate, calcium, and phosphorus.

In conclusion, several biological and mathematical factors have to be taken in consideration for the planning of a MPT as well as for its interpretation in order to the best possible answers. Great care has to be taken in chosing statistical methods as well as for establishing reference values, otherwise the impact of extreme values or outliers could lead to false interpretation. It is also important for most metabolites to aim the sampling at the group at risk, especially with regard to the stage of lactation. Comparison between samples over time have to take some seasonal variations into account. Finally, the site of venipunction of the database yielding the reference values has to be respected for one own's testing. 